\title{
Urothelial carcinoma in a pyelocaliceal diverticulum discovered by magnetic resonance urography
}

Jun Akatsuka, Yasutomo Suzuki, Tsutomu Hamasaki, Go Kimura, Yukihiro Kondo

Departments of Urology, Nippon Medical School, 1-1-5 Sendagi, Bunkyo-ku, Tokyo 113-8603, Japan

\section{ABSTRACT}

Neither computed tomography (CT) nor intravenous pyelography (IVP) alone can diagnose tumors of renal pelvic diverticula, but magnetic resonance urography (MRU) can obtain accurate preoperative information.

\section{CASE}

A 78-year-old woman presented with a history of macrohematuria for several months. Cystoscopy showed no bleeding from the left and right ureteral orifices or the bladder wall.

Figure 1 - Intravenous pyelography findings. Arrow shows left renal pelvic diverticulum.

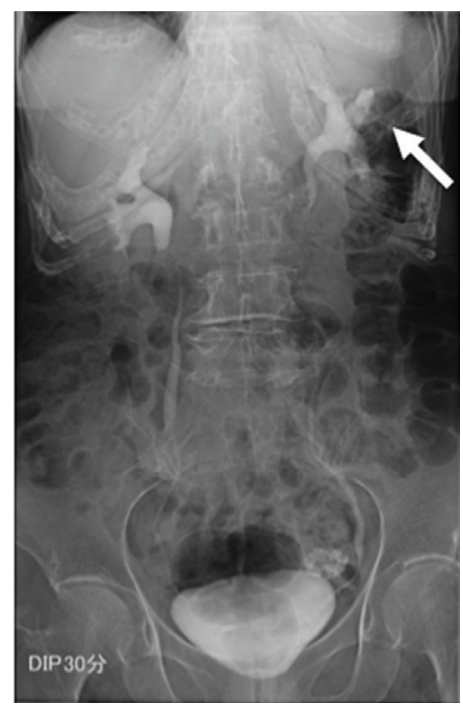

Urine cytology was class $\mathrm{V}$, and tumor markers including serum squamous cell carcinoma antigen and cyfra 21-1 were not elevated. The IVP, CT and MRU findings indicated a left renal pelvic diverticulum (Figure-1), left renal calcification (Figure-2) and a tumor in the pyelocaliceal diverticulum (Figure-3), respectively. Brain, chest, abdominal and pelvic CT did not detect distant metastasis. The tumor in the pyelocaliceal diverticulum was treated by left laparoscopic nephrouretero-cystectomy. The macroscopic findings showed a papillary tumor in

Figure 2 - Computed tomography findings. Left renal calcification is evident.

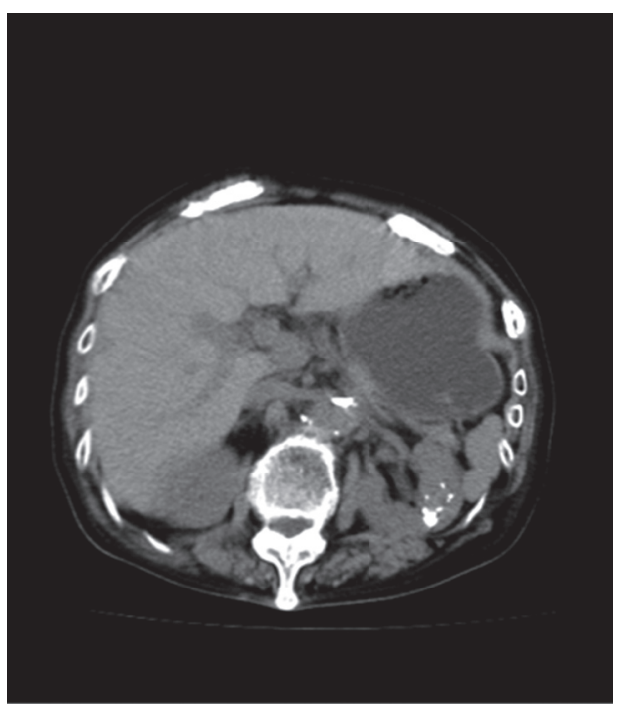


the pyelocaliceal diverticulum of the left kidney (Figure-4). The pathological findings revealed papillary urothelial carcinoma with squamous metaplasia of the left renal pelvic diverticulum, G2 >> G3, pT1, INFb, and negative surgical margins. The patient has remained alive without recurrence for 14 months.

Figure 3 - Magnetic resonance urography findings. Arrow shows $1.5-\mathrm{cm}$ mass in pyelocaliceal diverticulum.

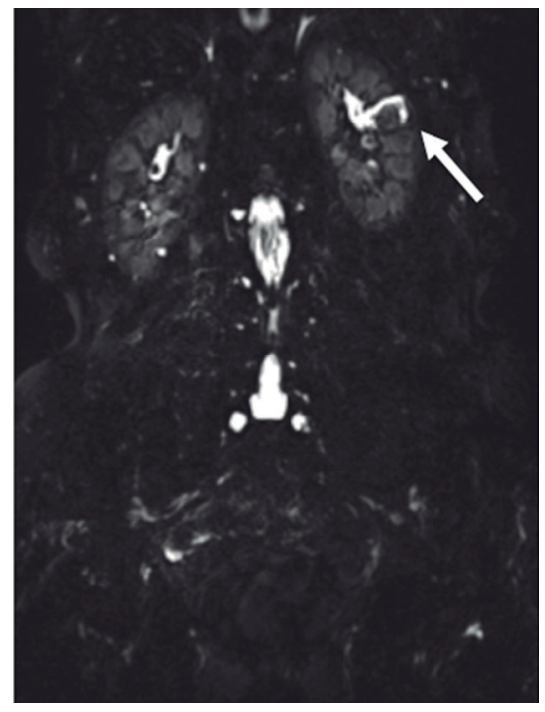

\section{DISCUSSION}

Pyelocaliceal diverticula are congenital, nonsecretory, urothelium-lined cavities within the renal parenchyma that communicate with the caliceal fornix through the diverticular neck. They occur at rates of $0.21 \%$ to $0.45 \%$ of excretory urograms (intravenous pyelograms) and calculi occur in $9.5 \%$ to $50 \%$ of all diverticula $(1,2)$. However, urothelial carcinoma of a pyelocaliceal diverticulum is exceedingly rare; fewer than 20 patients have been described in the literature since 1960 (3).

Pyelocaliceal diverticula that communicate with the caliceal fornix through the diverticular neck must be proven before tumors of renal pelvic diverticula can be diagnosed.

We finally diagnosed urothelial carcinoma of a pyelocaliceal diverticulum in this patient based on the MRU and pathological findings that fulfilled the diagnostic criteria. The pathological features of pyelocaliceal diverticulum tumors tend to resemble those of high-grade urothelial carcinoma (4). Several reports have described treatment by radical nephrectomy because of difficulties associated with preoperative diagnoses using only

Figure 4 - Macroscopic appearance of left kidney. Arrow shows tumor in pyelocaliceal diverticulum.

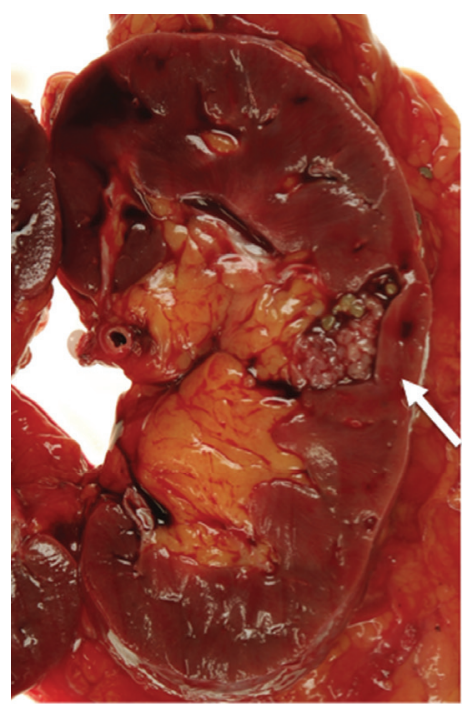

CT and IVP. Preoperative diagnosis is important, because tumors of pyelocaliceal diverticula should be treated like upper urothelial cancer, that is, by nephroureterectomy. Strict follow-up is required because the long-term prognosis is unknown. To our knowledge, this is the first report of a urothelial carcinoma of the pyelocaliceal diverticulum discovered by MRU. Urothelial carcinoma of the pyelocaliceal diverticulum is rare and often presents a diagnostic and therapeutic challenge. Magnetic resonance urography is helpful for diagnosing urothelial carcinoma of a pyelocaliceal diverticulum.

\section{ABBREVIATIONS}

$\mathrm{CT}=$ Computed tomography

IVP = Intravenous pyelography

MRU = Magnetic resonance urography 


\section{REFERENCES}

1. Middleton AW Jr, Pfister RC: Stone-containing pyelocaliceal diverticulum: embryogenic, anatomic, radiologic and clinical characteristics. J Urol. 1974; 111: 2-6.

2. Timmons JW Jr, Malek RS, Hattery RR, Deweerd JH: Caliceal diverticulum. J Urol. 1975; 114: 6-9.

\section{ARTICLE INFO}

Int Braz J Urol. 2014; 40: 274-6

Submitted for publication:

August 08, 2013

Accepted after revision:

September 09, 2013
3. Nishiura T, Yokoyama S: A case of transitional cell carcinoma and calcium in a pyelocalyceal diverticulum. Hinyouki Gakkaishi. 1961; 52:87-88.

4. Yoshimura K, Yoshida H, Kawase N, Taki Y: A case of transitional cell carcinoma and milk of calcium in a pyelocalyceal diverticulum. Hinyokika Kiyo. 1998; 44: 649-52.

\section{Correspondence address:}

Jun Akatsuka, MD

Department of Urology, Nippon Medical School

1-1-5 Sendagi, Bunkyo-ku, Tokyo 113-8603, Japan

Fax: +81 35 685-1794

E-mail: s00-001@nms.ac.jp 